\title{
Left Atrial Myxoma - A Case Report
}

\begin{abstract}
Cardiac tumours are rare in cardiovascular pathology but myxomas are the most common primary cardiac tumours, frequently found in left atrium, affected more women. Clinical presentation ranges from non specific symptoms to symptoms depending on size, location and mobility of the tumour. We present a case of asymptomatic left atrial myxoma in 50 years old female patient which was incidental finding. During the hospitalization we performed many investigations to confirm the diagnosis: ECG, laboratory examination, transthoracic echocardiography (TTE), transesophageal echocardiography (TEE), as well as coronary angiography. After confirming the diagnosis of cardiac tumour we transferred the patient to a surgical centre for further treatment. Pathohistological results after surgical removal of the tumour confirmed the diagnosis of cardiac myxoma. One year follow-up by echocardiography control showed that there are no signs of tumour recurrence and patient feels well.
\end{abstract}

Keywords: Atrial myxoma
Case Report

Volume 5 Issue 2 - 2016

Otljanska Magdalena* and Boshev Marjan

University Clinic of Cardiology, Medical Faculty, University "Sts. Cyril and Methodius", Macedonia

*Corresponding author: Otljanska Magdalena, University Clinic of Cardiology, Medical Faculty, University Campus

"Mother Theresa" - Skopje, Republic of Macedonia, Tel: +389

72231 006; Email: magdalenaotljanska@gmail.com

Received: December 13, 2015 | Published: January 29, 2016

\section{Introduction}

Atrial myxomas are the most common primary heart tumours and account for $40-50 \%$ of primary cardiac tumours. Because of nonspecific symptoms, early diagnosis may be a challenge. Twodimensional echocardiography is the diagnostic procedure of choice. Most atrial myxomas are benign and can be removed by surgical resection $[1,2]$.

Cardiac myxoma represents $30-50 \%$ of all benign tumours, $25 \%$ of all tumours and cysts of heart, usually are solitary and develop in the atria, $75 \%$ originating from the left atrium and $15-$ $20 \%$ from the right atrium. These tumours arise from or near the interatrial septum at the border of the fossa ovalis membrane and occur in all age groups, most frequently between the third and sixth decade. Myxomas are typically pedunculated with a stalk that is attached to the interatrial septum in fossa ovalis region. Women are more commonly affected and myxomas usually occur sporadically but there are also familiar forms. In about $20 \%$ cases, myxomas are asymptomatic and are discovered as an incidental finding $[3,4]$.

Myxomas are polypoid, round, or oval. Their clinical features are determined by their location, size, and mobility of myxoma. In left-sided myxomas symptoms more frequently occur when the diameter is over $5 \mathrm{~cm}$ or when the tumour reaches about 70 grams. Clinical presentation is categorised in four general manifestations - systemic, embolic, cardiac manifestations and phenomena secondary to metastatic diseases.

Symptoms range from non-specific constitutional to sudden cardiac death. Symptoms of intracardiac flow obstruction are the most commonly described manifestation, occurring in more than $50 \%$ of persons with cardiac myxomas. Typically, patients show symptoms of left-sided heart failure (eg. dyspnea, orthopnea, or fatigue) or syncope. Right atrial myxomas can manifest with symptoms of right-sided heart failure (eg, systemic oedema or hepatomegaly).

About $30-40 \%$ of individuals with cardiac myxomas experience embolic phenomena. Embolization appears to be much more likely in myxomas that are friable. Sites of embolization include the central nervous system (CNS), kidneys, extremities, and coronary arteries. Clinical manifestations of embolization are broad and depend on the tissues downstream of the embolus. Paradoxical embolism may occur in individuals with an anatomically patent foramen ovale [5-7]. Nonspecific constitutional symptoms have been reported in $20-60 \%$ of individuals with cardiac myxomas. Such symptoms may include fever, arthralgia, myalgia, and weight loss. Laboratory findings may include elevated erythrocyte sedimentation rate (33\%), normochromic anemia (15\%), and thrombocytopenia (5\%). Myxomas have been demonstrated to produce numerous growth factors and cytokines $[3,5]$.

\section{Case Presentation}

Our patient was 50 years old female who visited our clinic because of the high blood pressure (2-3 years duration, regularly treated with antihypertensive therapy). One day before hospitalization patient was diagnosed an intracavital mass in the left atrium on ehocardiographic examination, highly suspected for cardiac myxoma. Right upon detection, patient was admitted to our clinic for further examination. She was asymptomatic with stable vital signs and normal physical examination findings. During the hospitalization period we performed:

a) Laboratory examination - SER - $6 \mathrm{~mm} / \mathrm{h}$, hemoglobin $-141 \mathrm{~g} / \mathrm{L}$, $\mathrm{RBC}-4,5 \times 1012 / \mathrm{L}, \mathrm{WBC}-5,4 \times 109 / \mathrm{L}$, glucose 5,8 mmol/L, BUN - 3,4 mmol/L, creatinin - $49 \mu \mathrm{mol} / \mathrm{L}$, sodium - 139 $\mathrm{mmol} / \mathrm{L}$, potassium - 4,2 mmol/L, CRP - 4,0 mg/L,

b) Transthoracic and transesophageal echocardiography, 
c) Chest X-ray - with normal finding,

d) Coronary angiography - with no significant lesions of the coronary arteries,

e) ECG (Figure 1) - showed normal sinus rhythm with nonspecific changes.

Transthoracic echocardiogram (Figure 2 \& 3) revealed a large atrial mass occupying the majority of the left atrium with dimensions $2,97 \times 4,28 \mathrm{~cm}$ with no obstruction of the mitral valve orifice. Transesophageal echocardiography (Figure $4 \& 5$ ) confirmed presence of a large mass in the left atrium attached to the interatrial septum without prolaps of the tumour through the mitral valve. After coronary angiography and completion of preoperative dignostics, patient was transferred in another institution for surgical treatment. After surgical removal of the cardiac tumour, we received the histopathological material (Figure 6) which confirmed the diagnosis of cardiac myxoma. We continued with follow up of the patient by echocardiography control right after surgical treatment, as well as after 3 months, after 6 months, and after 12 months with no signs of tumor recurrence. She feels well and continues to take regularly antihypertensive drugs [7-9].

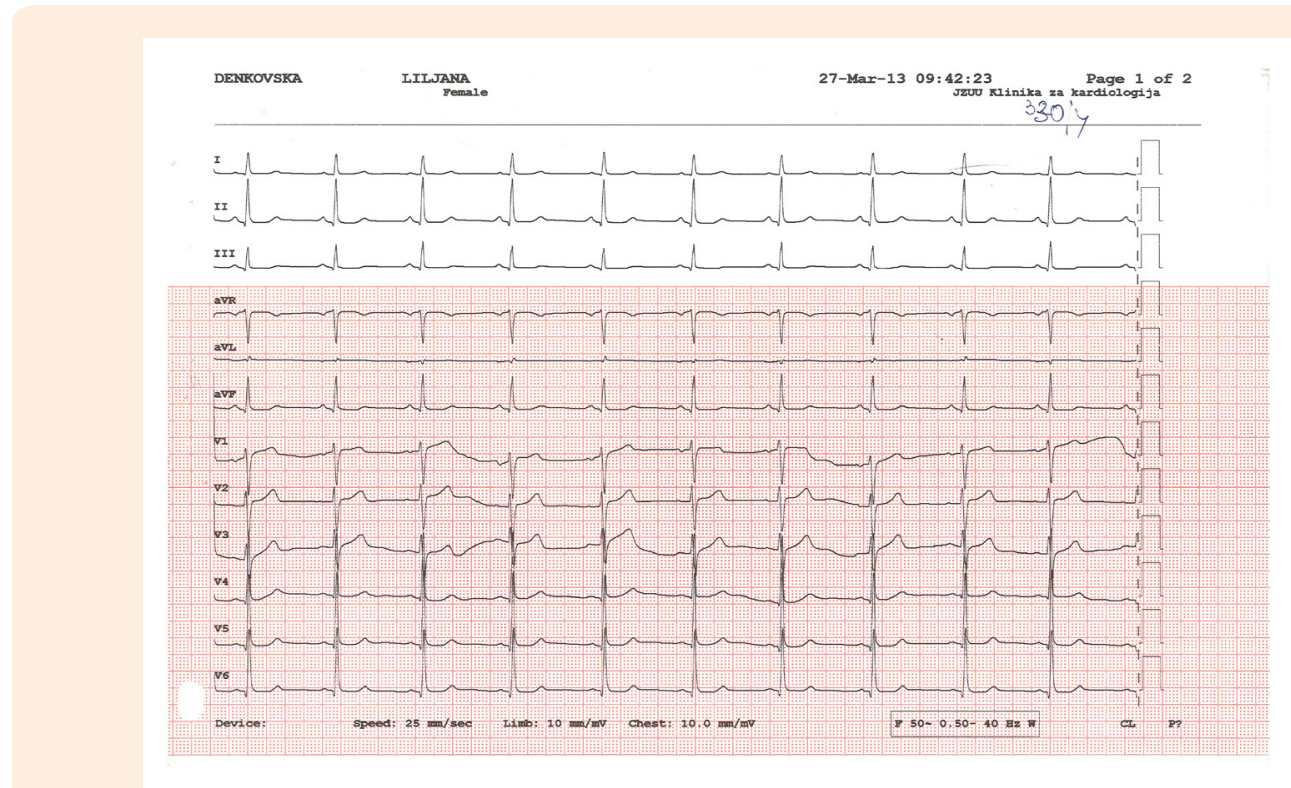

Figure 1: ECG showing sinus rhythm with nonspecific changes.

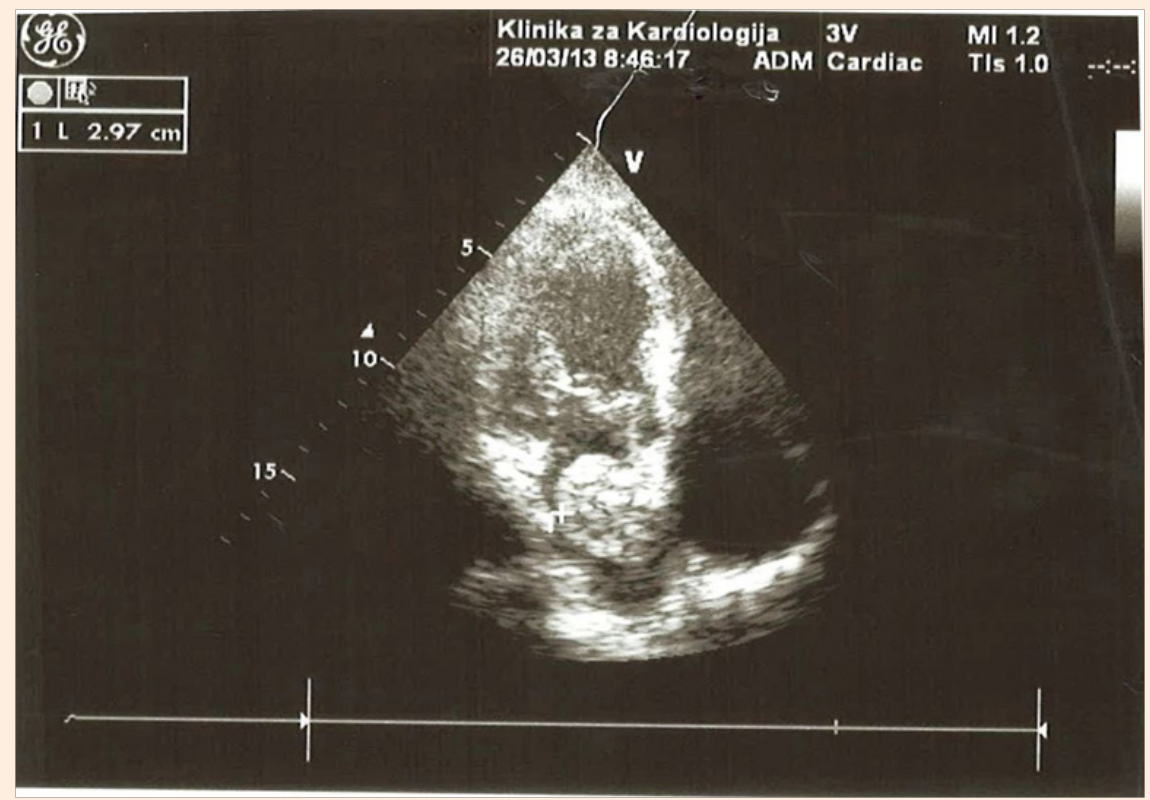

Figure 2: TTE showing large mass attached to the atrial septum. 


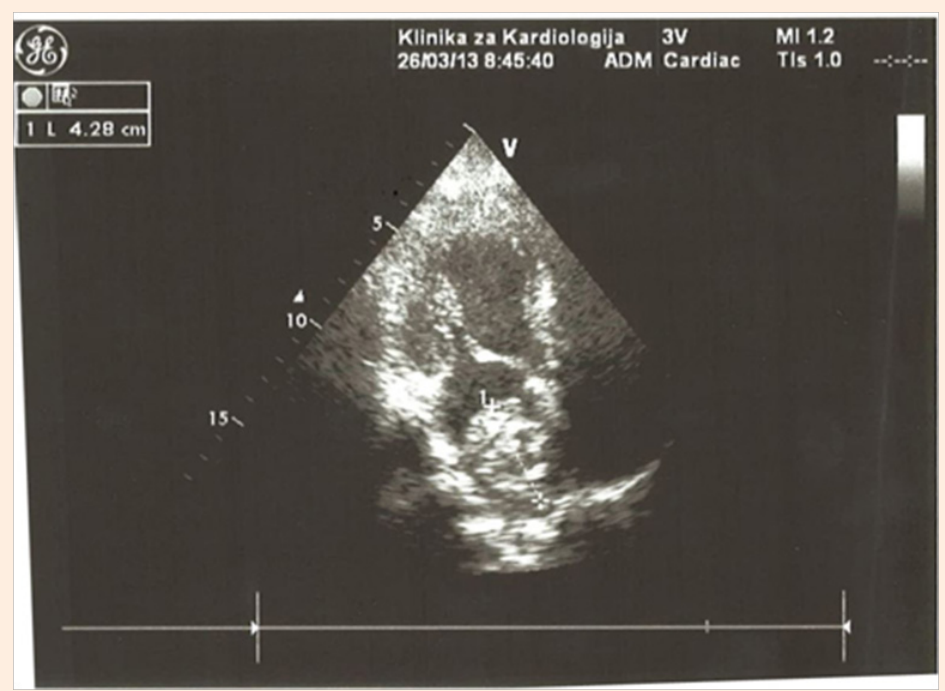

Figure 3: TTE showing large mass attached to the atrial septum (another position).

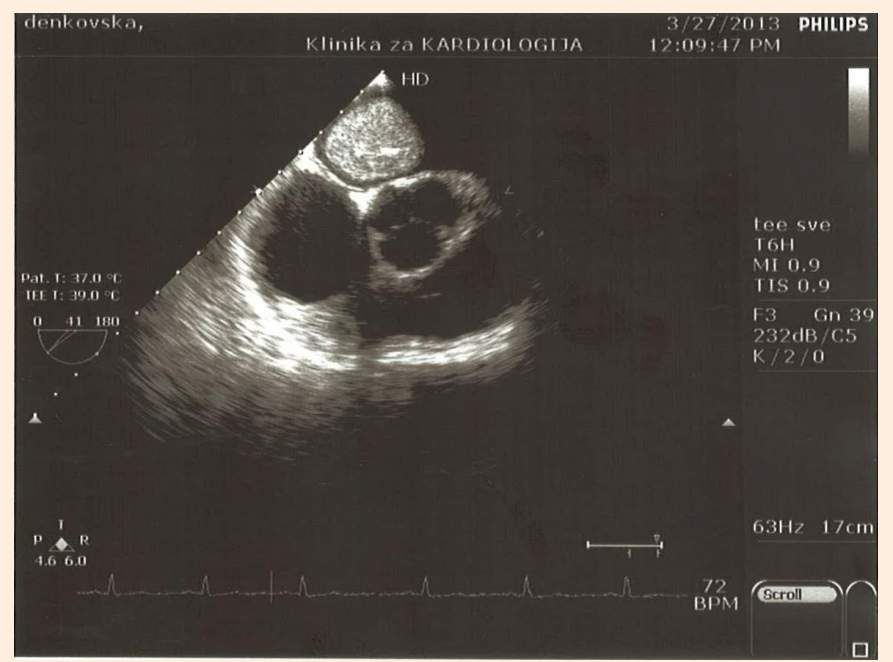

Figure 4: TEE image of the left atrial myxoma.

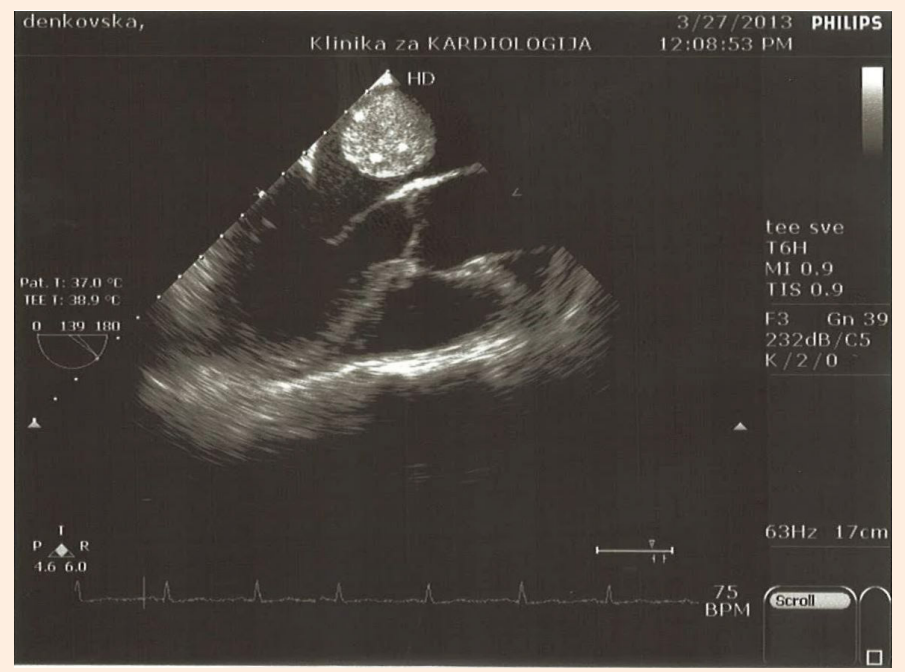

Figure 5: TEE image of the left atrial myxoma with no visible obstruction the mitral valve orifice. 

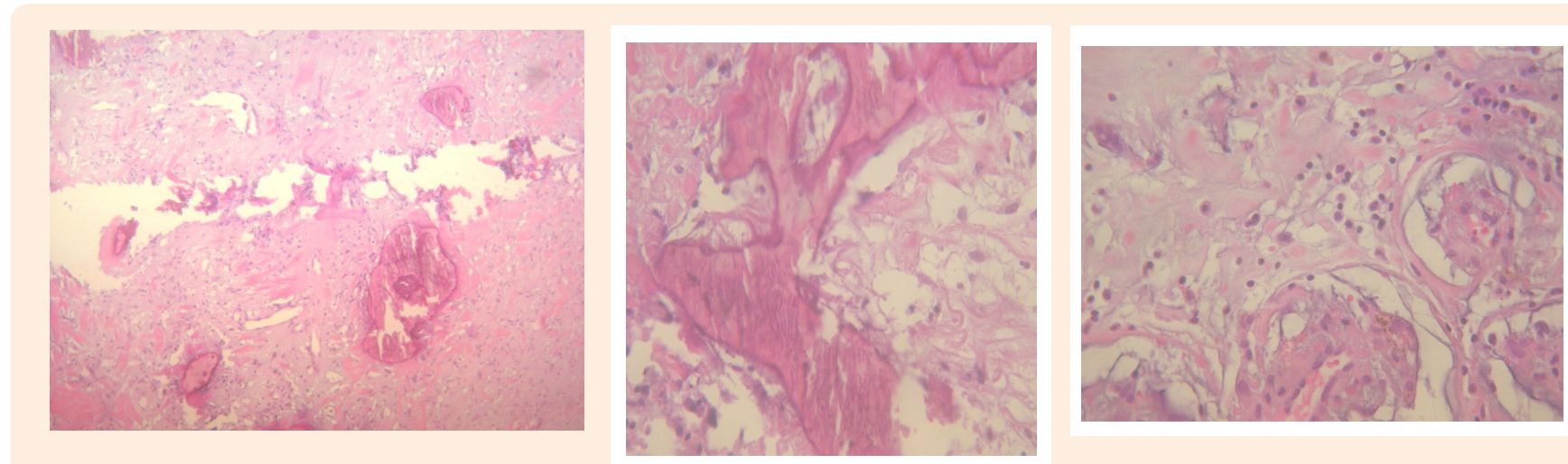

Figure 6: Pathohistological finding after surgical extraction of the left atrial myxoma.

\section{Discussion}

About $75 \%$ of myxomas originate from the left atrium and women are more commonly affected. Our patient was female and asymptomatic which can be usually found in about $20 \%$ of myxomas. Detection of left atrial mass was incidental finding. Size of myxoma with diameter of less than $5 \mathrm{~cm}$ can be an explanation for asymptomatic patient. ECG may show signs of left atrial enlargement, atrial fibrillation, atrial flutter, or conduction disturbances due to direct infiltration of cardiac conduction tissue or nonspecific changes as it was in our case. Chest X-ray was normal.

Diagnosis with two-dimensional echocardiography is usually adequate with good sensitivity detecting 95\% of myxomas but we can complete the diagnosis with transesophageal echocardiography as a more sensitive method [10-12]. For diagnosis of cardiac tumours we can also use cardiac MRI, computer tomography (CT) and PET scan. Coronary angiography is used to exclude co-existing coronary artery disease in patients over 40 or 50 years of age, depending on the surgical centre $[8,13]$.

For recurrence of cardiac myxoma it is very important to know whether the myxoma is familial. The recurrence rate is $1-3 \%$ in patients with sporadic myxomas but $10-20 \%$ in patients with familial myxomas. In patients with sporadic myxomas, incomplete excision is thought to be the most likely reason for tumour recurrence and clinical follow-up is recommended for individuals diagnosed with cardiac myxomas especially with familial cardiac myxomas.

Conventional treatment of atrial myxoma is surgical removal. Operative resection of the myxoma is the treatment of choice. A cloth patch or parietal pericardium is used to close the surgical defect. Damaged valves may require annuloplasty or prosthetic replacement in case of prolapse of a tumour through the mitral or tricuspid valve resulting in the destruction of the annulus or valve leaflets.

\section{References}

1. Sutsch G, Jenni R, von Segesser L, Schneider J (1991) Heart tumors: incidence, distribution, diagnosis. Exemplified by 20,305 echocardiographies. Schweizerische Medizinische Wochenschrift. 121(17): 621-629.
2. Burke A, Virmani R (1996) Atlas of Tumor Pathology. ( $3^{\text {rd }}$ edn.), Armed Forces Institute of Pathology, Tumors of the heart and great vessels, Washington, DC, USA, pp. 79-90.

3. Oliveira R, Branco L, Galrinho A, Abreu A, Abreu J, et al. (2010) Cardiac myxoma: a 13-year experience in echocardiographic diagnosis. Rev Port Cardiol 29(7-8): 1087-1100.

4. Bireta C, Popov AF, Schotola H, Trethowan B, Friedrich M, et al. (2011) Carney-Complex: multiple resections of recurrent cardiac myxoma. J Cardiothorac Surg 6: 12 .

5. Larsson S, Lepore V, Kennergren C (1989) Atrial myxomas: results of 25 years experience and review of the literature. Surgery 105(6): 695698.

6. Aggarwai SK, Barik R, Sarma TC, Lyer VR, Sai V, et al. (2007) Clinical presentation and investigation findings in cardiac mixoma: new insights from the developing world. Am Heart J 154(6): 1102-1107.

7. Pergolini A, Zampri G, Sbaraglia F, Musumeci F (2013) Left atrial myxoma:two sides of the coin. J Cardiovasc Med (Hagerstown) 16 Suppl 2: S74-76.

8. Paydarfar D, Krieger D, Dib N, Blair RH, Pastore J0, et al. (2001) In vivo magnetic resonance imaging and surgical histopathology of intracardiac masses: distinct features of subacute thrombi. Cardiology 95(1): 40-47.

9. Mugge A, Daniel WG, Haverich A, Lichtlen PR (1991) Diagnosis of noninfective cardiac mass lesions by two-dimensional echocardiography. Comparison of the transthoracic and transesophageal approaches. Circulation 83(1): 70-78.

10.Kühl HP, Hanrath P (2004) The impact of transesophageal echocardiography on daily clinical practice. Eur J Echocardiogr 5(6): 455-468.

11.Mulvagh SL, Rakowski H, Vannan MA, Abdelmoneim SS, Becher $\mathrm{H}$, et al. (2008) American society of echocardiography consensus statement on the clinical applications of ultrasonic contrast agents in echocardiography. J Am Soc Echocardiogr 21(11): 1179-1201.

12.Kaya H, Gökdeniz T, Tuncer A, Ozkan M (2010) Left atrial myxoma demonstrated by real-time three-dimensional transesophageal echocardiography. Turk Kardiyoloji Dernegi Arsivi 38(3): 222.

13.Gulati G, Sharma S, Kothari SS, Juneja R, Saxena A, et al. (2004) Comparison of echo and MRI in the imaging evaluation of intracardiac masses. Cardiovasc Intervent Radiol 27(5): 459-469. 